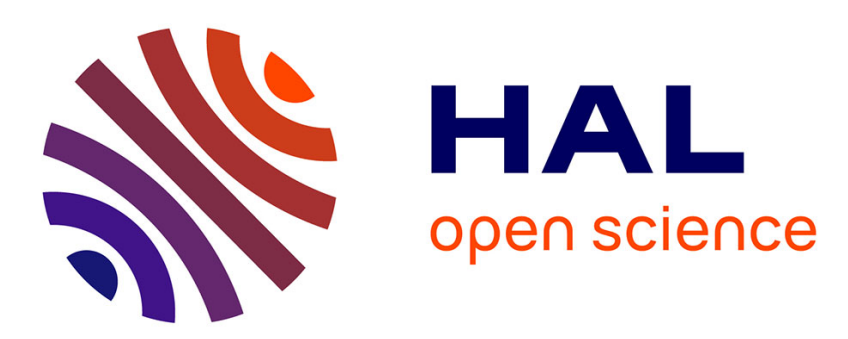

\title{
A Markovian Queueing System for Modeling a Smart Green Base Station
}

\author{
Ioannis Dimitriou, Sara Alouf, Alain Jean-Marie
}

\section{To cite this version:}

Ioannis Dimitriou, Sara Alouf, Alain Jean-Marie. A Markovian Queueing System for Modeling a Smart Green Base Station. EPEW: European Performance Evaluation Workshop, Aug 2015, Madrid, Spain. pp.3-18, 10.1007/978-3-319-23267-6_1 . hal-01215801

\section{HAL Id: hal-01215801 \\ https://hal.inria.fr/hal-01215801}

Submitted on 15 Oct 2015

HAL is a multi-disciplinary open access archive for the deposit and dissemination of scientific research documents, whether they are published or not. The documents may come from teaching and research institutions in France or abroad, or from public or private research centers.
L'archive ouverte pluridisciplinaire HAL, est destinée au dépôt et à la diffusion de documents scientifiques de niveau recherche, publiés ou non, émanant des établissements d'enseignement et de recherche français ou étrangers, des laboratoires publics ou privés. 


\title{
A Markovian queueing system for modeling a smart green base station
}

\author{
Ioannis Dimitriou ${ }^{1}$, Sara Alouf ${ }^{2}$, and Alain Jean-Marie ${ }^{23}$ \\ 1 Dept. of Mathematics, University of Patras, Greece, idimit@math.upatras.gr, \\ 2 Inria, Sophia Antipolis, France, Sara.Alouf@inria.fr, \\ 3 LIRMM, Montpellier, France, Alain.Jean-Marie@inria.fr
}

\begin{abstract}
We investigate a model to assess the performance of a base station (BS) fully powered by renewable energy sources. The BS is modeled as a three-queue system where two of them are coupled. One represents accumulated energy, the second is the data queue and the third one serves as a reserve energy queue. This smart BS is able to dynamically adjust its coverage area (thereby controlling the traffic intensity) and to generate signals to the reserve energy queue that trigger the movement of energy units to the main energy buffer. Given the randomness of renewable energy supply and the internal traffic intensity control, our queueing model is operated in a finite state random environment. Using the matrix analytic formalism we construct a five-dimensional Markovian model to study the performance of the BS. The stationary distribution of the system state is obtained and key performance metrics are calculated. A small numerical example illustrates the model and a simplified product-form approximation is proposed.
\end{abstract}

Keywords: Coupled queues, QBD processes, Green base station

\section{Introduction}

The architectural design of cellular networks has evolved in recent years to better satisfy users needs. The traffic load generated by users exhibits a night-day pattern, having a peak of traffic during the day and almost no traffic during the night. A geographical pattern is also observed as offices areas witness a peak traffic during the day while residential areas witness a smaller peak late in the evening. Heterogeneous cellular networks are an attractive deployment solution: large powerful base stations (BSs) are used to ensure coverage and connectivity whereas smaller coverage-limited BSs are used to accommodate the peak load where needed. Those BSs can be analyzed in isolation.

In this paper, we consider a single small BS and study the question of powering it using solely renewable energy. A key factor is that the solar radiation exhibits a night-day pattern that makes solar panels fit to power a small BS. We assume that the BS is "smart" in the sense that it is able to dynamically adjust its coverage area, controlling thereby the number of mobiles with which it communicates, and consequently its offered traffic rate and its energy consumption. The harvested energy is stored in batteries that are used to power the BS. 
We propose to model this BS as a queueing system that operates in a finitestate Markovian random environment (RE). The behavior of such a system is described by a five-dimensional Markov process, which is a homogeneous finite Quasi Birth-Death (QBD) process.

The literature on QBD processes is abundant and these processes have been used to study many different applications. De Cuypere et al. [4], have studied sensor nodes [3] and kitting processes through a paired queueing system [15]. In [3], a finite energy queue is paired with an infinite data queue, where customers arrive at both queues according to Markovian Arrival Processes. In [4], both queues are finite, but due to the sparsity of the generator matrix of the underlying Markov process, the size of the state space does not cause serious issues. Closely to our work, Takahashi et al. [17] consider a synchronization queue consisting of two buffers with finite capacities, the arrival processes at both buffers are Poisson for one and phase type renewal for the other. In our work we assume that energy is discretized (as in e.g. $[3,8,9]$ ) but it is also possible to model batteries as fluid queues; see for instance $[12,11]$.

In the following, we present our model in Section 2 and detail the infinitesimal generator of the five-dimensional continuous-time Markov chain representing the state of the system in Section 3. Section 4 discusses several algorithms that could be used to compute the stationary distribution of the system state and relevant performance metrics, exploiting the QBD structure of the generator. The feasibility of the developed algorithm is demonstrated through a numerical example. First passage times to lower and higher levels in the QBD are discussed in Section 5. In Section 6, we propose an approximate model with product-form solution. We briefly conclude in Section 7.

\section{The model}

We are interested in a wireless communications base station (BS), isolated from the electric grid and operating thanks to renewable energy sources. The model supposes a continuous functioning of the station: it does not handles issues like startup, shutdown, malfunction and other transitory phenomena.

The model we develop has a 5-dimensional state space, representing the state of three queues, a service process and an environment process. We describe now these elements and the way they interact. Figure 1 summarizes the model under study. Although we describe here a quite specific situation, we point out that all these elements and their composition can be generalized so as to build models of other device configurations.

Queues. We consider two energy queues (EQ) of finite capacity $E_{j}, j=1,2$ that store energy extracted from the environment, and a data queue (DQ) that keeps track of packets not yet transmitted, having also a buffer of finite capacity $N$. Energy is assumed to be discretized, as for instance in $[8,9]$. Assume that EQ 1, is coupled with the DQ, whereas EQ 2 is deployed close to the BS and serves as a reserve. Each EQ, is fed by different renewable energy sources, and the choice of the one that will be coupled with the DQ depends on the area where the BS 


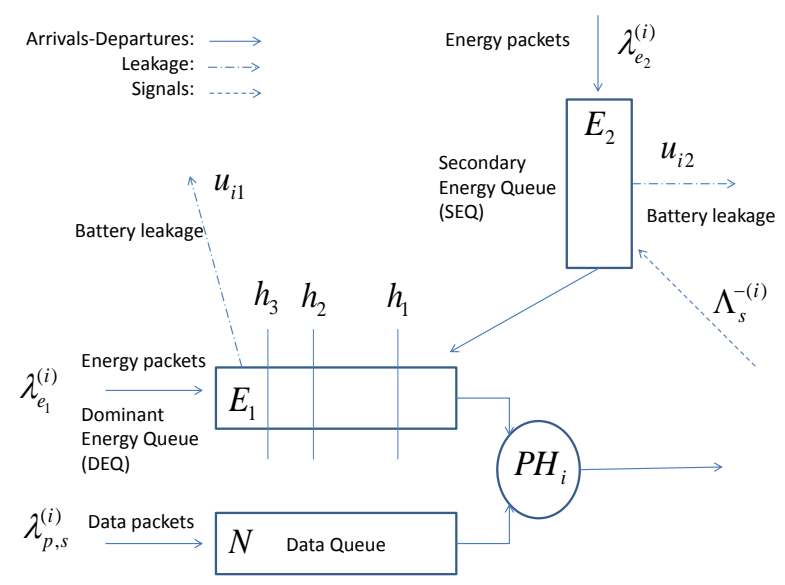

Fig. 1. The model when the RE is in state $i$.

is deployed. We will refer to this EQ, say EQ 1, as the dominant energy queue (DEQ) and the EQ 2 as the secondary energy queue (SEQ).

Environment. The system behavior depends on the state of the RE. This RE is defined by means of an irreducible regular continuous-time Markov chain $Y(t)$ with state space $\{1, \ldots, M\}$, and infinitesimal generator $Q_{Y}$. The state of this environment may represent a variety of factors that make energy arrival or packet arrival processes, energy consumption and service times non time-stationary. For instance, the intermittent nature of wind, the variability of sunlight, can be taken into account with an environment variable. Hourly variations of data traffic can also be modeled that way. Other features can be the variation of transmission power due to global adjustments at the network level. As usual, independent environment features can be combined in a complex environment process with multiple "phases", at the cost of large values for $M$. Clearly, one can skip the $\mathrm{RE}$ and reduce the dimension of the QBD, by assuming that the model migrates quickly from the stationary situation of one environment setting to another.

Packet service duration. The service time distribution is of phase type $[16,14]$ of order $\nu$ and depends on the state of the RE. For a fixed RE state $i$, this distribution can be interpreted as a time until some underlying Markov process $\eta_{t}$ with finite state space $\{1, \ldots, \nu\}$ reaches the absorbing state 0 with initial probability vector $\left(\tau_{0}^{(i)}, \underline{\tau}^{(i)}\right)$. Transition rates of the process $\eta_{t}$ within the set $\{1, \ldots, \nu\}$ are defined by the sub-generator $T^{(i)}$ and transition rates into the absorbing state (which lead to a service completion) are given by the entities of the column vector $\underline{t}^{(i)}=-T^{(i)} \underline{1}$. Observe that the value $\nu$ does not depend on $i$. A typical situation allowed by this model is to have a packet size with some given distribution, and a service speed given by the environment: $T_{k l}^{(i)}=T_{k l} \times v_{i}$, $v_{i}$ begin the "velocity" typical of state $i$ and $T_{k l}$ some environment-independent 
transition rate. If the packet length distribution itself can also depend on the environment, the model may not be accurate for the packet in service during the change of environment state.

By using a generalized PH distribution for the service process, we can incorporate several realistic concepts of the operation of a BS such as: different levels of noise in the transmission channel, hardware degradation and recovering, variations in the distance of a mobile user to the base station, etc.

Packet arrival. The rate of packet arrival to the BS depends on its coverage area. We adopt a multi-threshold scheme in order for the BS to dynamically adjust the coverage area according to the available energy units in the DEQ: in this sense, we model a smart BS. More precisely, we introduce thresholds, say $h_{0}=0<h_{1}<$ $h_{2}<\ldots<h_{K}<E_{1}=h_{K+1}$. Given the state $i$ of RE, and if $h_{s}<m_{1} \leq h_{s+1}$, $s=0, \ldots, K$, the users' arrival rate equals $\lambda_{p, s}^{(i)}$. In practice, it is expected that $\lambda_{p, 0}^{(i)} \leq \lambda_{p, 1}^{(i)} \leq \ldots \leq \lambda_{p, K}^{(i)}$ but this is not needed for the definition of the model. Note that the packet traffic potentially includes system control packets, signaling (e.g. for handovers) etc. Superposition of different traffic sources can classically be taken care of.

Energy arrival and depletion. For a fixed state $i, i=1, \ldots, M$ of the RE, energy units are stored in EQ $j$ at rate $\lambda_{e_{j}}^{(i)}$. Transmission opportunities occurs only when both the DEQ and the DQ queue are non empty.

The modeling of energy consumption by a packet transmission requires some trick. Indeed, it is unlikely that a phase of the service of some packet will consume exactly an integer amount of energy quanta. Randomness in consumption and/or non-integral energy values can be taken into account using random variables.

The number of energy units required for the transmission of a single packet depends both on the state of the RE and on the phase of the service process. Thus, the transmission of a packet may be interrupted, if the completion of a phase of service consumes the available energy. In this paper, we assume that the transmission of this packet has to restart from scratch (but with a stochastically independent value), whenever there will be available energy. If some mechanism, e.g. error-correcting codes, allows for it, transmission could resume from the same phase. Alternately, the transmission of the packet could be simply canceled in case of energy shortage. Moreover, we can also allow this cancellation to occur only after some timeout, by introducing a timer for each "impatient" packet when energy is 0 . None of these alternatives would make the transition matrix much more complex. With the "restart" point of view, given the state $i$ of RE and if there are $m_{1}$ available energy units in the DEQ, the completion of phase $x$, $x=1,2, \ldots, \nu$ requires $k$ energy units with probability $p_{k x}^{\left(i, m_{1}\right)}, \sum_{k=0}^{m_{1}} p_{k x}^{\left(i, m_{1}\right)}=1$. We allow a priori $k=0$, i.e. that the completion of a phase of service may not consume even a single energy unit. However, in order to initiate a transmission we need at least one energy unit.

Energy leakage from each EQ is unavoidable. More precisely, an energy unit will be lost from EQ $j, j=1,2$ at exponential rate $u_{i j}$, given that the RE is in state $i$. Note that if the DEQ drains during the transmission of a packet, the packet has to be retransmitted, whenever there will be available energy units. 
Energy transfers. The SEQ serves as backup storage for the DEQ. The control of our smart BS generates signals to the SEQ at a rate $\Lambda_{s}^{-(i)}$, that trigger the movement of energy units to the DEQ, according to the multi-threshold rule introduced above. Given the state $i$ of the RE, and $h_{s}<m_{1} \leq h_{s+1}, s=0, \ldots, K$, signals are generated at a rate $\Lambda_{s}^{-(i)}$, and trigger [7] $k$ energy units from the SEQ towards the DEQ with probability $q_{k s}^{\left(m_{1}, m_{2}, i\right)}\left(\sum_{k=1}^{m_{2}} q_{k s}^{\left(m_{1}, m_{2}, i\right)} \delta_{m_{1}+k \leq E_{1}}=\right.$ 1). Define also $q_{k s}^{\left(m_{1}, m_{2}\right)}=\operatorname{diag}\left(q_{k s}^{\left(m_{1}, m_{2}, 1\right)}, \ldots, q_{k s}^{\left(m_{1}, m_{2}, M\right)}\right)$. In case the SEQ is empty, the signal will have no effect. As above, it is expected in practice that $\Lambda_{0}^{-(i)} \geq \Lambda_{1}^{-(i)} \geq \ldots \geq \Lambda_{K}^{-(i)}$ : the demand rate for replenishment of DEQ becomes larger as the energy in the DEQ depletes. It is also possible to reflect the urgency of replenishment by modifying the distribution $q_{k s}$. Moreover, when the DEQ is full, i.e. $m_{1}=E_{1}$, there will be no signal towards the SEQ (i.e., $\Lambda_{K}^{-(i)}=0$ ). We also adopt an overflow operation in the sense that when an EQ buffer is full, its energy traffic is rerouted to the other EQ. Clearly, the signal generation possibly consumes an amount of energy, which is assumed to be negligible in our model.

Thanks to the generality of the definition of the probabilities $p_{k x}^{\left(i, m_{1}\right)}, q_{k s}^{\left(m_{1}, m_{2}, i\right)}$ (which are function of the state of the RE, the phase of service process and the available energy in DEQ, SEQ), the modeler can incorporate additional realistic features regarding the energy consumption, related to the control channel data transmission, or processing and forwarding of packets. More complex packet processing architectures may however require more than one packet queue.

Clearly, recent technological developments are towards smart autonomic wireless networks. The concept of signaling towards the SEQ, that triggers the instantaneous transition of energy units to the DEQ, has become an intelligent modeling tool for communication systems. Queues with signals [7] were introduced to model the behavior of control actions such as the displacement of units from one queue to another using "triggers" resulting in load balancing.

\section{Process of the system state}

The behavior of the system under consideration can be described in terms of the CTMC $X_{t}=\left\{Q_{p}(t), J(t), Q_{e_{1}}(t), Q_{e_{2}}(t), Y(t)\right\}, t \geq 0$, where $Q_{p}(t), J(t), Q_{e_{j}}(t)$ and $Y(t)$ are, respectively, the number of data packets, the phase of the service process (present only when $Q_{p}>0$ and $Q_{e_{1}}>0$ ), the number of energy units in EQ $j$ and the state of RE at time $t$. The state space of $X_{t}$ is $\widehat{H}=\cup_{n=0}^{N} l(n)$ and the "levels" $l(n)$ are defined as:

$$
l(0)=\left\{\left(0, m_{1}, m_{2}, i\right) ; m_{j}=0,1, \ldots, E_{j}, j=1,2, i=1, \ldots, M\right\},
$$

and for $1 \leq n \leq N$ :

$$
\begin{aligned}
l(n)= & l(n, 0) \cup \bar{l}(n), \\
l(n, 0)= & \left\{\left(n, 0, m_{2}, i\right) ; m_{2}=0,1, \ldots, E_{2}, i=1, \ldots, M\right\}, \\
\bar{l}(n)= & \left\{\left(n, x, m_{1}, m_{2}, i\right) ; x=1, \ldots, \nu ; m_{1}=1, \ldots, E_{1} ; m_{2}=0,1, \ldots, E_{2} ;\right. \\
& i=1, \ldots, M\} .
\end{aligned}
$$


These levels have cardinals $|l(0)|=L_{0}:=M\left(E_{1}+1\right)\left(E_{2}+1\right)$ and for $n \geq 1$, $|l(n)|=L:=M\left(E_{2}+1\right)\left(\nu E_{1}+1\right)$. For convenience, define also $S:=M\left(E_{2}+1\right)$. The state space has then cardinal $|\widehat{H}|=M\left(E_{2}+1\right)\left[N\left(\nu E_{1}+1\right)+\left(E_{1}+1\right)\right]$.

Using this decomposition in levels, the infinitesimal generator of $X_{t}$ has a quasi-birth-death structure with block matrix representation as:

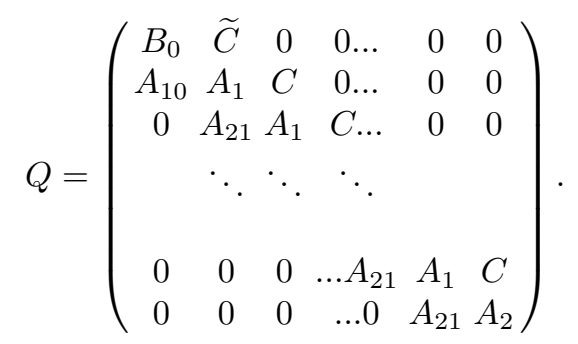

We proceed with a detailed description of the blocks. We shall need the definition of the following matrices $\Lambda_{e_{j}}=\operatorname{diag}\left(\lambda_{e_{j}}^{(i)}\right), j=1,2, \Lambda_{p, s}=\operatorname{diag}\left(\lambda_{p, s}^{(i)}\right)$, $\Lambda_{s}^{-}=\operatorname{diag}\left(\Lambda_{s}^{-(i)}\right), s=0, \ldots, K, U_{j}=\operatorname{diag}\left(u_{i j}\right)$, where $i=1, \ldots, M$, and $t_{x}=$ $\operatorname{diag}\left(t_{x}^{(1)}, \ldots, t_{x}^{(M)}\right), p_{l x}^{\left(m_{1}\right)}=\operatorname{diag}\left(p_{l x}^{\left(1, m_{1}\right)}, \ldots, p_{l x}^{\left(M, m_{1}\right)}\right)$.

The edge block $A_{10}$ is an $L \times L_{0}$ matrix that corresponds to packet service and energy consumption events that lead to an empty data queue. It has a blocktriangular structure with $A_{10}^{\left(0, m_{1}^{\prime}\right)}=0_{S \times S}$, for all $m_{1}^{\prime}$ (this first row corresponds to the case where there is a packet waiting for service, but the transmission cannot be initiated since there is no available energy in the DEQ), $A_{10}^{\left(m_{1}, m_{1}^{\prime}\right)}=0_{\nu S \times S}$ for $1 \leq m_{1}<m_{1}^{\prime} \leq E_{1}$ and for $m_{1}=1, \ldots, E_{1}, m_{1}^{\prime}=0, \ldots, m_{1}$ :

$$
\begin{aligned}
& A_{10}^{\left(m_{1}, m_{1}^{\prime}\right)}=A_{m_{1} m_{1}^{\prime}}^{\left(m_{1}-m_{1}^{\prime}\right)}= \\
& \left(I_{\left(E_{2}+1\right) \times\left(E_{2}+1\right)} \otimes\left[t_{1}^{\left(m_{1}\right)} p_{m_{1}-m_{1}^{\prime}, 1}^{\left(m_{m_{1}}\right)}\right], \ldots, I_{\left(E_{2}+1\right) \times\left(E_{2}+1\right)} \otimes\left[t_{\nu}^{\left(m_{1}\right)} p_{m_{1}-m_{1}^{\prime}, \nu}^{\left(m_{1}\right)}\right]\right)^{\prime} .
\end{aligned}
$$

The sub-diagonal block $A_{21}$ is an $L \times L$ matrix that corresponds to packet service and energy consumption:

$$
A_{21}=\left(\begin{array}{cccccc}
0_{S \times S} & 0_{S \times S} & 0_{S \times S} & \ldots & 0_{S \times S} & 0_{S \times S} \\
A_{10}^{(1)} & F_{11} & 0_{\nu S \times S} & \ldots & 0_{\nu S \times S} & 0_{\nu S \times S} \\
A_{20}^{(2)} & F_{21} & F_{22} & \ldots & 0_{\nu S \times S} & 0_{\nu S \times S} \\
\ldots & \ldots & \ldots & \ddots & & \\
\ldots & \ldots & \ldots & \ddots & & \\
A_{E_{1} 0}^{\left(E_{1}\right)} & F_{E_{1} 1} & F_{E_{1} 2} & \ldots & F_{E_{1} E_{1}-1} & F_{E_{1} E_{1}}
\end{array}\right)
$$

where $F_{m_{1} m_{1}^{\prime}}$ is a $\nu S \times \nu S$ matrix defined from $\tau_{x}=\operatorname{diag}\left(\tau_{x}^{(1)}, \ldots, \tau_{x}^{(M)}\right)$ as:

$$
F_{m_{1} m_{1}^{\prime}}=I_{\left(E_{2}+1\right) \times\left(E_{2}+1\right)} \otimes\left(t_{x} \tau_{y} p_{m_{1}-m_{1}^{\prime} x}^{\left(m_{1}\right)}\right), x, y=1, \ldots, \nu .
$$


Packet arrivals are represented in block $\widetilde{C}=\operatorname{diag}\left(C_{0}, C_{1}, \ldots, C_{K}\right)$, which is an $L_{0} \times L$ matrix where $C_{s}$ is of order $n(s) S \times n(s) S \nu$ for $s=1,2, \ldots, K$, and of order $n(0) S \times[(n(0)-1) \nu+1] S$ for $s=0$, with $n(s)=h_{s+1}-h_{s}+1 \delta_{s=0}$ and

$$
\begin{array}{r}
C_{0}=\operatorname{diag}(C_{00}, \underbrace{G_{0}, \ldots, G_{0}}_{h_{1}}), C_{s}=I_{n(s) \times n(s)} \otimes G_{s}, s=1, \ldots, K, \\
G_{s}=\left(G_{s}^{(1)}, \ldots, G_{s}^{(\nu)}\right), G_{s}^{(x)}=I_{\left(E_{2}+1\right) \times\left(E_{2}+1\right)} \otimes\left(\Lambda_{p, s} \tau_{x}\right) .
\end{array}
$$

Matrix $C=\operatorname{diag}\left(C_{0}^{\prime}, C_{1}^{\prime}, \ldots, C_{K}^{\prime}\right)$ is of order $L \times L$, where

$$
\begin{aligned}
C_{0}^{\prime}=\operatorname{diag}(C_{00}, \underbrace{G_{0}^{\prime}, \ldots, G_{0}^{\prime}}_{h_{1}}), C_{s}^{\prime}=I_{n(s) \times n(s)} \otimes G_{s}^{\prime}, s=1, \ldots, K, \\
G_{s}^{\prime}=I_{\nu \times \nu} \otimes C_{0 s}, C_{0 s}=I_{\left(E_{2}+1\right) \times\left(E_{2}+1\right)} \otimes \Lambda_{p, s}, s=0, \ldots, K .
\end{aligned}
$$

Next, $B_{0}=\left(B_{m_{1}, m_{1}^{\prime}}^{(0)}\right), 0 \leq m_{1}, m_{1}^{\prime} \leq E_{1}$, is an $L_{0} \times L_{0}$ matrix formed of square sub-blocks $B_{m_{1}, m_{1}^{\prime}}^{(0)}$ of order $S$, typical of energy movements in the DEQ that are independent of packet transmissions, such as energy leakage, energy generation and signal-triggered energy unit transition from SEQ to DEQ. Its structure is:

$$
B_{0}=\left(\begin{array}{cccccc}
B_{00}^{(0)} & B_{01}^{(0)} & B_{02}^{(0)} & \cdots & & B_{0 E_{1}}^{(0)} \\
\widehat{U}_{1} & B_{11}^{(0)} & B_{12}^{(0)} & \cdots & & B_{1 E_{1}}^{(0)} \\
\cdots & \cdots & \cdots & \cdots & & \\
& & & & & \\
0_{L_{0} \times L_{0}} & 0_{L_{0} \times L_{0}} & 0_{L_{0} \times L_{0}} & \ldots \widehat{U}_{1} & B_{E_{1}-1, E_{1}-1}^{(0)} & B_{E_{1}-1, E_{1}}^{(0)} \\
0_{L_{0} \times L_{0}} & 0_{L_{0} \times L_{0}} & 0_{L_{0} \times L_{0}} & \ldots 0_{L_{0} \times L_{0}} & \widehat{U}_{1} & B_{E_{1} E_{1}}^{(0)}
\end{array}\right)
$$

where $\widehat{U}_{1}=I_{\left(E_{2}+1\right) \times\left(E_{2}+1\right)} \otimes U_{1}$ represents energy leakage from DEQ, and for $0 \leq m_{1}<E_{1}, h_{s}<m_{1} \leq h_{s+1}$,

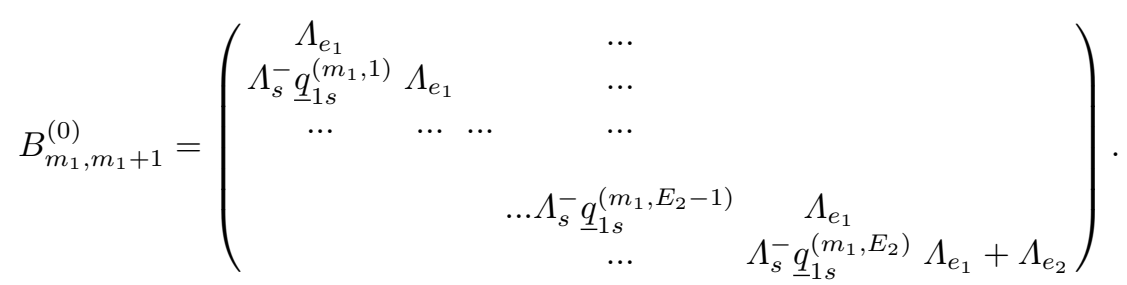

The remaining sub-blocks concern the energy replenishment due to signals. Assuming $E_{1} \leq E_{2}$, for $k=m_{1}+2, \ldots, E_{1}, h_{s}<m_{1} \leq h_{s+1}$,

$$
B_{m_{1} k}^{(0)}=\left(\begin{array}{ccccc} 
& & & & \\
\ldots & \ldots & \ldots & \ldots & \ldots \\
\Lambda_{s}^{-} \underline{q}_{k-m_{1} s}^{\left(m_{1}, k-m_{1}\right)} & & & & \ldots \\
& \Lambda_{s}^{-} \underline{q}_{k-m_{1} s}^{\left(m_{1}, k-m_{1}+1\right)} & & & \ldots \\
\ddots & \ddots & \ddots & \ddots & \\
& & \ldots \Lambda_{s}^{-} \underline{q}_{k-m_{1} s}^{\left(m_{1}, E_{2}\right)} & \ldots
\end{array}\right) .
$$


Remark: In this work we assume without loss of generality that $E_{1} \leq E_{2}$. For $E_{1}>E_{2}, k=m_{1}+2, \ldots, m_{1}+E_{2}, B_{m_{1} k}^{(0)}$ has also the same form but $B_{m_{1} k}^{(0)}=0_{S \times S}$, for $k=m_{1}+E_{2}+1, \ldots, E_{1}$.

Furthermore, for $0 \leq m_{1} \leq E_{1}$ and $h_{s}<m_{1} \leq h_{s+1}$, set $F_{s}=Q_{Y}-$ $\sum_{j=1}^{2}\left(\Lambda_{e_{j}} \delta_{m_{j}<E_{j}}+U_{j} \delta_{m_{j}>0}\right)-\Lambda_{p, s}-\Lambda_{s}^{-} \delta_{m_{1}<E_{1}}$. Then for $m_{2}, m_{2}^{\prime}=0, \ldots, E_{2}$, $B_{m_{1} m_{1}}^{(0)}=\left(B_{m_{1} m_{1}}^{\left(0 ; m_{2}, m_{2}^{\prime}\right)}\right)$ where, $B_{m_{1} m_{1}}^{\left(0 ; m_{2}, m_{2}-1\right)}=U_{2}$ and,

$$
B_{m_{1} m_{1}}^{\left(0 ; m_{2}, m_{2}+1\right)}=\Lambda_{e_{2}}+\Lambda_{e_{1}} \delta_{m_{1} \neq E_{1}}, \quad B_{m_{1} m_{1}}^{\left(0 ; m_{2}, m_{2}\right)}=F_{s}, h_{s}<m_{1} \leq h_{s+1} .
$$

Finally, the main diagonal blocks $A_{1}$ and $A_{2}$ are square matrices of order $L$, also composed of sub-blocks $A_{l}=\left(A_{m_{1} m_{1}^{\prime}}^{(l)}\right), m_{1}, m_{1}^{\prime}=0,1, \ldots, E_{1}$, for $l=1,2$. Here, $A_{00}^{(l)}$ is of order $S \times S, A_{0 m_{1}^{\prime}}^{(l)}, m_{1}^{\prime}=1, \ldots, E_{1}$ are of order $S \times \nu S, A_{m_{1} 0}^{(l)}$, $m_{1}=1, \ldots, E_{1}$, of order $\nu S \times S$, and $A_{m_{1} m_{1}^{\prime}}^{(l)}, m_{1}, m_{1}^{\prime}=1, \ldots, E_{1}$ of order $\nu S \times \nu S$.

They are given by, $A_{00}^{(1)}=B_{00}^{(0)}$ and $A_{0 m_{1}}^{(1)}=\left(Z_{1}^{\left(0, m_{1}\right)}, \ldots, Z_{\nu}^{\left(0, m_{1}\right)}\right)$ where $Z_{x}^{\left(0, m_{1}\right)}=\left(Z_{x ; m_{2}, m_{2}^{\prime}}^{\left(0, m_{1}\right)}\right), x=1, \ldots, \nu$, are $S \times S$ matrices where, $Z_{x ; m_{2}, m_{2}}^{(0,1)}=\left(\Lambda_{e_{1}}+\right.$ $\left.\Lambda_{e_{2}} \delta_{m_{2}=E_{2}}\right) \tau_{x}, m_{2}=0,1, \ldots, E_{2}, Z_{x ; m_{2}, m_{2}-1}^{(0,1)}=\Lambda_{1}^{-} \underline{q}_{11}^{\left(0, m_{2}\right)} \tau_{x}, Z_{x ; m_{2}, m_{2}^{\prime}}^{(0,1)}=0_{M \times M}$, elsewhere. For $m_{1}=2, \ldots, E_{1}, h_{s}<k \leq h_{s+1}$,

$$
Z_{x ; m_{2}, m_{2}^{\prime}}^{\left(0, m_{1}\right)}=\Lambda_{s}^{-} \underline{q}_{m_{1} s}^{\left(0, m_{2}\right)} \tau_{x}, m_{2}=m_{1}, \ldots, E_{2}, m_{2}^{\prime}=0,1, \ldots, m_{2}-m_{1},
$$

and $Z_{x ; m_{2}, m_{2}^{\prime}}^{\left(0, m_{1}\right)}=0_{M \times M}$ elsewhere. Furthermore, for $l=1,2, m_{1}=1, \ldots, E_{1}$, $A_{m_{1} 0}^{(l)}=\left(\widehat{S}_{m_{1} 1}, \ldots, \widehat{S}_{m_{1} \nu}\right)^{\prime}$, where

$$
\begin{aligned}
\widehat{S}_{m_{1} x}= & I_{\left(E_{2}+1\right) \times\left(E_{2}+1\right)} \otimes\left[U_{1} \delta_{m_{1}=1}\right. \\
& \left.+\operatorname{diag}\left(\sum_{c=1, c \neq x}^{\nu} T_{x c}^{(1)} p_{m_{1} x}^{\left(1, m_{1}\right)}, \ldots,+\sum_{c=1, c \neq x}^{\nu} T_{x c}^{(M)} p_{m_{1} x}^{\left(M, m_{1}\right)}\right)\right], \\
A_{m_{1} k}^{(l)}= & I_{\nu \times \nu} \otimes B_{m_{1} k}^{(0)}, k=m_{1}+1, \ldots, E_{1}, m_{1} \geq 1 .
\end{aligned}
$$

Moreover, $A_{m_{1}, m_{1}-1}^{(l)}=\left(A_{m_{1}, m_{1}-1}^{(l ; x, y)}\right), k=1,2, \ldots, \nu$, and $A_{m_{1}, m_{1}-1}^{(l ; x, x)}=\widehat{U}_{1}, x=$ $1,2, \ldots, \nu, A_{m_{1}, m_{1}-1}^{(l ; x, y)}=I_{\left(E_{2}+1\right) \times\left(E_{2}+1\right)} \otimes\left[T_{x y} p_{1 x}^{\left(m_{1}\right)}\right], x, y=1,2, \ldots, \nu, x \neq y$.

For $k=m_{1}-2, \ldots, 1, A_{m_{1} k}^{(l)}=\left(A_{m_{1} k}^{(l ; x, y)}\right), x, y=1, \ldots, \nu$, where, $A_{m_{1} k}^{(l ; x, x)}=0_{S \times S}$, $x=1, \ldots, \nu$, and $A_{m_{1} k}^{(l ; x, y)}=I_{\left(E_{2}+1\right) \times\left(E_{2}+1\right)} \otimes T_{x y} p_{m_{1}-k x}^{\left(m_{1}\right)}, x, y=1, \ldots, \nu, x \neq y$ and $A_{m_{1} k}^{(2)}=A_{m_{1} k}^{(1)}, k=m_{1}+1, \ldots, E_{1}$.

Moreover, $A_{m_{1} m_{1}}^{(l)}=\left(\Phi_{x y ; m_{2}, m_{2}^{\prime}}^{\left(l, m_{1}, m_{1}\right)}\right), l=1,2, x, y=1, \ldots, \nu, m_{l}=1, \ldots, E_{l}$, where for $T_{x y}=\operatorname{diag}\left(T_{x y}^{(1)}, \ldots, T_{x y}^{(M)}\right)$,

$$
\begin{aligned}
\Phi_{x y ; m_{2}, m_{2}^{\prime}}^{\left(l, m_{1}, m_{1}\right)} & =I_{\left(E_{2}+1\right) \times\left(E_{2}+1\right)} \otimes\left[T_{x y} p_{0 x}^{\left(m_{1}\right)}\right], m_{1}=0,1, \ldots, E_{1}, x \neq y, \\
\Phi_{x x ; m_{2}, m_{2}}^{\left(l, m_{1}, m_{1}\right)} & =I_{\left(E_{2}+1\right) \times\left(E_{2}+1\right)} \otimes\left(T_{x x}+\Lambda_{p, s} \delta_{m_{1}<E_{1}}\right)+B_{m_{1} m_{1}}^{(0)}+W_{s} \delta_{m_{1}<E_{1}}, \\
W_{s} & =\operatorname{diag}\left(0_{M \times M}, I_{\left(M E_{2} \times M E_{2}\right)} \otimes \Lambda_{s}^{-}\right) .
\end{aligned}
$$


Sparsity of the generator. The number of non-zero transition rates in the generator $Q$ can be roughly evaluated as follows. Neglecting the "border" cases, transitions from a typical state $\left(n, x, m_{1}, m_{2}, i\right)$ occur as: a) packet arrival, energy arrival, energy leakage: one transition each; b) service phase change: $\nu-1$ possible transitions with service continuation, plus service completion, which triggers energy consumption with up to $m_{1}$ possible transitions; c) environment phase change: $M-1$ possible transitions (this could be much less if the phase process is itself a superposition, see Section 2); d) energy transfers: up to $m_{2}$ transitions. Summing over $m_{1}$ and $m_{2}$, we conclude that storing the elementary blocks of $Q$ requires $O\left((\nu+M) M E_{1}^{2} E_{2}^{2}\right)$ space. Storing all $Q$ in sparse form would require $O\left(N(\nu+M) M E_{1}^{2} E_{2}^{2}\right)$ space. The ratio of this quantity with the total number of entries in $Q$ : $O\left(\left(N M \nu E_{1} E_{2}\right)^{2}\right)$, is of order $\alpha=O((\nu+M) / N \nu M)$. It may even be smaller if the RE transition matrix $Q_{Y}$ is itself sparse, or if the distributions $p_{k x}^{\left(i, m_{1}\right)}$ or $q_{k s}^{\left(m_{1}, m_{2}, i\right)}$ have restricted supports. Note that some algorithms work with storing only the different blocks.

\section{Stationary solution}

\subsection{Algorithms for stationary probabilities}

Under reasonable assumptions on packet arrival rates and the representation of service distributions, the chain $X_{t}$ is irreducible. Since it is finite, it then admits a unique stationary distribution given by (referring to the notation in (1) and $(2))$ :

$$
\begin{gathered}
p\left(n, x, m_{1}, m_{2}, i\right) \\
=\lim _{t \rightarrow \infty} P\left(Q_{p}(t)=n, J(t)=x, Q_{e_{1}}(t)=m_{1}, Q_{e_{2}}(t)=m_{2}, Y(t)=i\right) \\
p\left(n, m_{1}, m_{2}, i\right)=\lim _{t \rightarrow \infty} P\left(Q_{p}(t)=n, Q_{e_{1}}(t)=m_{1}, Q_{e_{2}}(t)=m_{2}, Y(t)=i\right) .
\end{gathered}
$$

In order to compute this stationary probability vector, several methods are available. On the one hand, the QBD structure of $Q$ offers the possibility to use direct numerical methods, such as the Matrix Analytic ones. Those are "exact" (up to round off errors) and in general they are efficient especially for systems where their generator matrix has a special form, which is the case here. On the other hand, the sparsity of $Q$ points at the use of iterative and other advanced techniques [18].

One way or the other, a closer investigation of the block structure of $Q$ is fruitful. The stationary distribution vector $p$ is represented using the level structure as $\underline{p}=\left(\underline{p}_{0}, \underline{p}_{1}, \ldots, \underline{p}_{N}\right)$. Global balance equations $\underline{p} Q=0$ can then be written as

$$
\begin{aligned}
\underline{p}_{0} B_{0}+\underline{p}_{1} A_{10} & =0 \\
\underline{p}_{0} \widetilde{C}+\underline{p}_{1} A_{1}+\underline{p}_{2} A_{21} & =0, \\
\underline{p}_{i-1} C+\underline{p}_{i} A_{1}+\underline{p}_{i+1} A_{21} & =0, \quad i=2, \ldots, N-1, \\
\underline{p}_{N-1} C+\underline{p}_{N} A_{2} & =0 .
\end{aligned}
$$


The matrix analytic formalism gives the opportunity to obtain the stationary probability vector $p$ using several algorithms (see [14] and references within, and later contributions among which $[1,5,19])$. The methods of $[6,19]$ are highlighted in [14, Chap. 10]. The key step in [6] resides especially in the inversion of a matrix of order $L_{0}$ and $N$ matrices of order $L$. Given that $L_{0} \leq L$, the overall complexity is $O\left(N L^{3}\right)$. The "folding method" in [19] is less expensive, reducing the " $N$ " to $\log _{2} N$, but at a higher implementation cost. Moreover, [6] also provides an algorithm for the computation of the expected first passage times between the neighbour levels. This result is interesting for further capacity planning investigation, see Section 5 and our conclusion. In our situation, the limiting factor is clearly the value of $L \approx M \nu E_{1} E_{2}$.

The form of the block generator matrix will help us to improve the efficiency in obtaining the stationary probabilities, since the matrix $C$ is a diagonal nonsingular matrix. Solving recursively system (11)-(14) starting from (14) we get that, $\underline{p}_{n}=\underline{p}_{N} R_{n}$ for $n=0, \ldots, N$, with: $R_{N}=I, R_{N-1}=-A_{2} C^{-1}, R_{0}=$ $\left(R_{2} A_{1}+R_{3} A_{21}\right) A_{10} B_{0}^{-1}$, and

$$
R_{n}=-\left(R_{n+1} A_{1}+R_{n+2} A_{21}\right) C^{-1}, n=1, \ldots, N-2 .
$$

To obtain $\underline{p}_{N}$, we solve equation (12) along with the normalizing condition:

$$
\underline{p}_{N}\left(R_{0} \widetilde{C}+R_{1} A_{1}+R_{2} A_{21}\right)=0, \quad \underline{p}_{N}\left(R_{0} \underline{1}_{0}+\sum_{n=1}^{N} R_{n} \underline{1}\right)=1,
$$

where $\underline{1}, \underline{1}_{0}$ are column vectors of order $L$ and $L_{0}$ respectively.

The computation of the $R_{n}$ 's involves only matrix additions and multiplications. Since $C$ is diagonal and non singular, multiplying by its inverse is not costly. The computation of the $p_{n}$ 's [5] requires two matrix inversions (one to compute $B_{0}^{-1}$, one to solve the system (16)), $4 N+5$ matrix multiplications and $2 N+1$ matrix additions. The resulting complexity is $O\left(L^{3}\right)$. Using an iterative method to solve (16) and exploiting the inner structure of blocks (see Section 2) may help overcome difficulties about the computational complexity.

\subsection{Performance metrics}

By calculating the stationary distribution vector of the underlying Markov process we can obtain some important performance metrics, such as the depletion probability $(D P)$ (i.e., the probability of an empty DEQ), and the expected number of data packets and energy packets in each EQ:

$$
\begin{aligned}
& D P= \sum_{n=0}^{N} \sum_{m_{2}=0}^{E_{2}} \sum_{i=1}^{M} p\left(n, 0, m_{2}, i\right), \\
& E\left(Q_{p}\right)=\sum_{n=1}^{N} \sum_{m_{2}=0}^{E_{2}} \sum_{i=1}^{M} n\left[p\left(n, 0, m_{2}, i\right)\right.\left.+\sum_{m_{1}=1}^{E_{1}} \sum_{x=1}^{\nu} p\left(n, x, m_{1}, m_{2}, i\right)\right], \\
& E\left(Q_{e_{1}}\right)=\sum_{m_{1}=1}^{E_{1}} \sum_{m_{2}=0}^{E_{2}} \sum_{i=1}^{M} m_{1}\left[p\left(0, m_{1}, m_{2}, i\right),\right. \\
&\left.+\sum_{n=1}^{N} \sum_{x=1}^{\nu} p\left(n, x, m_{1}, m_{2}, i\right)\right],
\end{aligned}
$$




$$
\begin{aligned}
E\left(Q_{e_{2}}\right)=\sum_{m_{2}=1}^{E_{2}} \sum_{i=1}^{M} m_{2}\left[\sum_{m_{1}=0}^{E_{1}} p\left(0, m_{1}, m_{2}, i\right)\right. \\
\left.\quad+\sum_{n=1}^{N}\left(p\left(n, 0, m_{2}, i\right)+\sum_{m_{1}=1}^{E_{1}} \sum_{x=1}^{\nu} p\left(n, x, m_{1}, m_{2}, i\right)\right)\right] .
\end{aligned}
$$

Using Little's law we can obtain the mean waiting time for a data packet to be served. Moreover, various optimization problems can be formulated, such as finding optimal values of $\lambda_{p, s}^{(i)}$ that minimizes $E\left(Q_{p}\right)$, asking $D P \leq a$, or finding an optimal value for $E_{1}$ which minimizes $D P=D P\left(E_{1}\right)$.

\subsection{Numerical example}

To demonstrate the feasibility of the developed algorithm and numerically show some features of the model, we present the results of a preliminary numerical experiment, inspired from values taken from [13]. Assume that the RE is defined by the 2 -state infinitesimal generator with rates $\left(Q_{Y}\right)_{12}=0.01$ and $\left(Q_{Y}\right)_{21}=$ 0.1 . We may interpret that the first state of the RE corresponds to a mode when the system is overloaded by the data units (peak time) and the second state to a normal mode of the system. Under the first and second state of the RE the service time distribution is characterized by the vectors $\underline{\tau}^{(1)}=(0.2,0.8)$, $\underline{\tau}^{(2)}=(0.7,0.3)$ and the matrices

$$
T^{(1)}=\left(\begin{array}{cc}
-0.4368 & 0.40768 \\
0.42608 & -1.71809
\end{array}\right), \quad T^{(2)}=\left(\begin{array}{cc}
-0.6402 & 0.56812 \\
0.22308 & -1.34325
\end{array}\right)
$$

respectively. We assumed for $m_{1}+m_{2} \leq E_{1}, q_{k s}^{\left(m_{1}, m_{2}, i\right)}=2^{k i} / \sum_{j=1}^{m_{2}} 2^{j i}, k=$ $1, \ldots, m_{2}$ and if $m_{1}+m_{2}>E_{1}, q_{k s}^{\left(m_{1}, m_{2}, i\right)}=2^{k i} / \sum_{j=1}^{E_{1}-m_{1}} 2^{j i}, k=1, \ldots, E_{1}-m_{1}$. Furthermore, $p_{k x}^{\left(i, m_{1}\right)}=\left(\sum_{j=1}^{m_{1}}(i x)^{k-j}\right)^{-1}, k=1, \ldots, m_{1}$. Table 1 contains the values of the parameters of the system.

Table 1. Overview of system's parameters

\begin{tabular}{c|c|c|c}
\hline \hline$E_{1}=10, E_{2}=12$ & $M=2, \nu=2$ & $N=15$, & $h_{1}=8, h_{2}=12$ \\
\hline$\Lambda_{p, 1}=\operatorname{diag}(1.5,1)$ & $\Lambda_{p, 2}=\operatorname{diag}(2,1.5)$ & $U_{1}=\operatorname{diag}(0.1,0.3)$ & $U_{2}=\operatorname{diag}(0.3,0.4)$ \\
\hline$\Lambda_{1}^{-}=\operatorname{diag}(0.3,0.2)$ & $\Lambda_{2}^{-}=\operatorname{diag}(0.2,0.1)$ & $L_{e_{1}}=\operatorname{diag}(1.5,1)$ & $L_{e_{2}}=\operatorname{diag}(1.8,1.2)$ \\
\hline \hline
\end{tabular}

We focus on the depletion probability. Figure 2 (left) describes the way the depletion probability is affected by the $\mathrm{RE}$ for increasing values of the data arrival rate when $m_{1} \in[0,8](s=0)$. Clearly, the depletion probability will increase, but this increase will become more apparent during the overloaded period, as expected. In Figure 2 (right) we can observe the benefits of the smart operation of the base station due to the presence of signals. By increasing the signal generation rate, the depletion probability is strongly reduced. As a result, the quality of service is thoroughly increased. 

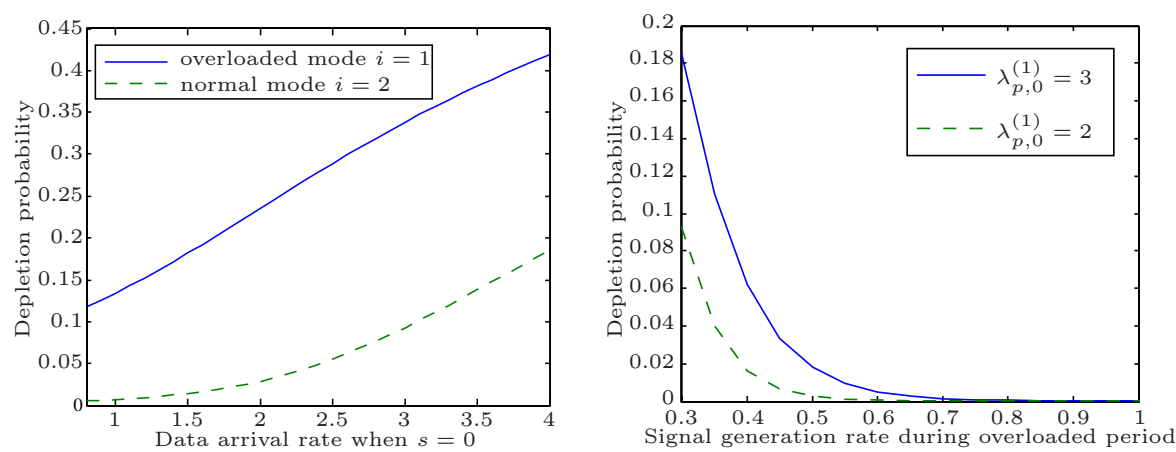

Fig. 2. Sensibility of DP w.r.t. data rate and RE for $\Lambda_{0}^{-}=\operatorname{diag}(0.35,0.25)$ (left) and w.r.t. signal rate when the $\mathrm{RE}$ is in overload

\section{First passage times}

Gaver et al. [6] analyzed the first passage times for general finite QBD processes. They obtained two systems of recurrence equations for the Laplace-Stieltjes transforms of passage times to higher and lower levels.

In the following we turn our attention to the computation of the average first passage times. Let the expected values of the first passage time from the states of $l(n-1)$ to the states of $l(n)$ be $c^{(n)}$ and that from the states of $l(n+1)$ to $l(n)$ be $v^{(n)}$. Define the following matrices:

$$
\begin{array}{rlrl}
H_{0} & =B_{0} & H_{1}=A_{1}+A_{10}\left(-H_{0}^{-1}\right) \widetilde{C}, \\
H_{j} & =A_{1}+A_{21}\left(-H_{j-1}^{-1}\right) C, & 2 \leq j \leq N-1, \\
H_{N} & =A_{2}+A_{21}\left(-H_{N-1}^{-1}\right) C . & &
\end{array}
$$

Then we have the following recurrence relations for the expected values of first passage times to higher levels:

$$
c^{(n)}= \begin{cases}-H_{0}^{-1} \underline{1}, & n=1, \\ -H_{1}^{-1}\left(\underline{1}+A_{10} c^{(1)}\right), & n=2, \\ -H_{n-1}^{-1}\left(\underline{1}+A_{21} c^{\left(m_{1}-1\right)}\right), & 3 \leq n \leq N .\end{cases}
$$

For the determination of the expected values of the first passage time to lower levels we have first to denote by $\widehat{H}_{n}, n=0,1, \ldots, N$ the infinitesimal generator of the restriction of the process $X_{t}$ observed during those intervals of time spent at $l(n)$ before the original process moves to $l(n-1)$ for the first time. Then, all $\widehat{H}_{n}$-processes, $1 \leq n \leq N$ are transient while $\widehat{H}_{0}$ is positive recurrent. The matrices $\widehat{H}_{n}$ are recursively computed as follows

$$
\widehat{H}_{n}= \begin{cases}B_{0}+\widetilde{C}\left(-\widehat{H}_{1}^{-1}\right) A_{10}, & n=0 \\ A_{1}+C\left(-\widehat{H}_{n+1}^{-1}\right) A_{21}, & 1 \leq n \leq N-1 \\ H_{N}, & n=N\end{cases}
$$


Then,

$$
v^{(n)}= \begin{cases}-\widehat{H}_{1}^{-1}\left(\underline{1}+\widetilde{C} v^{(1)}\right), & n=0, \\ -\widehat{H}_{n+1}^{-1}\left(\underline{1}+C v^{(n+1)}\right), & 1 \leq n \leq N-2, \\ -\widehat{H}_{N}^{-1} \underline{1}, & n=N-1 .\end{cases}
$$

These computations can be exploited, for instance, for controlling the (expected) time at which the packet queue overflows for the first time. Based on them, values for the control thresholds $h_{s}$ and packet arrival rates $\lambda_{p, s}^{(i)}, s=0 . . K$ can be determined numerically offline. If the computation is fast enough, online adaptation becomes possible. Another use of first passage times is to compute the average length of busy periods. From this metric, analyses about the opportunity to put the BS in sleep mode during idle periods can be envisioned.

Computing first passage times for energy levels is also of great interest, in order to determine and optimize the autonomy of the BS. However, the model we have presented is not directly a QBD for energy levels, and a more elaborate analysis is the topic of a work in progress.

\section{$6 \quad$ An approximated product-form model}

In this section, we describe a simplification of the model proposed in Section 2, strongly motivated by recent works in $[8,9]$. This new model is a G-network and therefore has product-form solution. The simplification applies to instances of our model where: a) there is no threshold control for the DEQ, therefore just environment-dependent packet as well as signal arrival rates $\lambda_{p}^{(i)}, \Lambda^{-(i)}, i=1 . . M$; b) exactly one energy unit is required to transmit any data packet.

Consider the following simplifications: 1) infinite capacity buffers for the DEQ, SEQ, and the data queue; 2) as soon as the BS has both a data packet and enough energy to transmit that packet, the transmission is instantaneous; 3 ) environment-dependent parameters are averaged out using the stationary distribution $\underline{q}_{Y}$ of the generator $Q_{Y}$. Thus, defining the vector $\underline{\lambda}_{p}=\left(\lambda_{p}^{(1)}, \ldots, \lambda_{p}^{(M)}\right)$ and $\underline{\Lambda}^{-}=\left(\Lambda^{-(1)}, \ldots, \Lambda^{-(M)}\right)$, then the arrival rates for packets, DEQ and SEQ energy units, and signals in the simplified model are as:

$$
\widehat{\lambda}_{p}=\underline{\lambda}_{p} \underline{q}_{Y}^{\prime}, \quad \widehat{\lambda}^{-}=\underline{\Lambda}^{-} \underline{q}_{Y}^{\prime}, \quad \widehat{\lambda}_{e_{j}}=\underline{1}_{e_{j}} \underline{q}_{Y}^{\prime}, j=1,2 .
$$

Similarly, we will take into account the average leakage rate for each energy queue, i.e., if $\underline{u}_{j}=\underline{1} U_{j} \underline{q}_{Y}^{\prime}, j=1,2$, where $\underline{1}$ is an $1 \times M$ vector of 1 .

The state of the approximation model can be represented by the pair $(n, m)$, where $n=0$, means that the BS has neither energy units in the DEQ, nor data packets to transmit, while $n>0$, means that it currently stores $n$ data packets but no energy units in DEQ, while $n<0$, means that it stores $-n$ energy units in DEQ but no data packets. The element $m \geq 0$, counts the number of stored energy units in SEQ. Note that our system is a special type of G-networks. The underlying Markov process $\widetilde{X}=\left\{(n, m) ; n \in Z, m \in Z^{+} \cup\{0\}\right\}$ is ergodic 
provided that:

$$
\widehat{\lambda}_{p}<\widehat{\lambda}_{e_{1}}+\widehat{\lambda}^{-} q_{2}, \quad \widehat{\lambda}_{e_{2}}<\widehat{u}_{2}+\widehat{\lambda}^{-}, \quad \widehat{\lambda}_{e_{1}}+\widehat{\lambda}^{-} q_{2}<\widehat{\lambda}_{p}+\widehat{u}_{1},
$$

where $q_{2}=\widehat{\lambda}_{e_{2}} /\left(\widehat{u}_{2}+\hat{\lambda}^{-}\right)$, is the probability that the SEQ is not empty. These conditions come from the fact that the system must be stable with respect to the data packets $(n>0$, left hand condition), with respect to the energy units in SEQ, (the condition at the middle), and with respect to the energy units in DEQ $(n<0$, right hand condition). To conclude we state the following proposition.

Proposition 1. Under conditions (S) and with $f(m)=q_{2}^{m}, m \geq 0$, the joint stationary distribution of $\widetilde{X}$ has the product form:

$$
\begin{gathered}
p(n, m)=C g(n) f(m), \\
g(0)=1, \quad g(n)=q_{1}^{n}, n>0, \quad g(n)=\left(\tilde{q}_{1}\right)^{-n}, n<0, \\
q_{1}=\frac{\widehat{\lambda}_{p}}{\widehat{\lambda}_{e_{1}}+\widehat{\lambda}^{-} q_{2}}, \quad \tilde{q}_{1}=\frac{\widehat{\lambda}_{e_{1}}+\widehat{\lambda}^{-} q_{2}}{\widehat{\lambda}_{p}+\widehat{u}_{1}}, \quad C=\frac{\left(1-q_{1}\right)\left(1-\tilde{q}_{1}\right)\left(1-q_{2}\right)}{1-q_{1} \tilde{q}_{1}} .
\end{gathered}
$$

The proof proceeds with the substitution of (21) into the global balance equations, and is omitted due to page constraint. For alternative approaches see $[2$, 10]. $C$ is obtained using the normalizing condition $\sum_{n=-\infty}^{+\infty} \sum_{m=0}^{+\infty} p(n, m)=1$.

Extension to a network of $S E Q$ s. The approximation model is flexible enough to incorporate an arbitrary number of SEQs that are deployed close to the target BS while preserving the product-form solution. Indeed, assume that there are $K$ reserve energy queues deployed close to our BS. In such a case our smart BS will generate signals at SEQ $k$, at a Poisson rate $\widehat{\lambda}_{k}^{-}$, while energy units arrive at SEQ $k$ at a Poisson rate $\widehat{\lambda}_{e_{k}}^{r}$, and energy leakage at a Poisson rate $\widehat{u}_{2 k} k=1, \ldots, K$. The system's state is now $(n, \underline{m})=\left(n, m_{1}, \ldots, m_{K}\right), n \in Z, m_{k}=0,1, \ldots$, where now $m_{k}$, counts the number of stored energy units in SEQ $k$.

Then, provided that $\check{q}_{1}=\frac{\widehat{\lambda}_{p}}{\widehat{\lambda}_{e_{1}}+\sum_{k=1}^{K} \widehat{\lambda}_{k}^{-} q_{2 k}}<1, \check{\tilde{q}}_{1}=\frac{\widehat{\lambda}_{e_{1}}+\sum_{k=1}^{K} \widehat{\lambda}_{k}^{-} q_{2 k}}{\widehat{\lambda}_{p}+\widehat{u}_{1}}<1$, $q_{2 k}=\frac{\widehat{\lambda}_{e_{k}}^{r}}{\widehat{\lambda}_{k}^{-}+\widehat{u}_{2 k}}<1, k=1, \ldots, K$, a modification of Proposition 1 can be proved, and the join stationary distribution of $(n, \underline{m})$ is for $f_{k}\left(m_{k}\right)=q_{2 k}^{m_{k}}, k=1, \ldots, K$, $m_{k} \geq 0$,

$$
\begin{gathered}
p(n, \underline{m})=C^{\prime} v(n) \prod_{k=1}^{K} f_{k}\left(m_{k}\right), \\
v(0)=1, \quad v(n)=\check{q}_{1}^{n}, n>0, \quad v(n)=\left(\tilde{\tilde{q}}_{1}\right)^{-n}, n<0,
\end{gathered}
$$

and the normalization constant is $C^{\prime}=\left(1-\check{q}_{1}\right)\left(1-\tilde{q}_{1}\right) \prod_{k=1}^{K}\left(1-q_{2 k}\right) /\left(1-q_{1} \tilde{q}_{1}\right)$.

\section{Conclusion}

We have given the detailed specification of a versatile model of energy supply for base stations or similar devices and presented preliminary numerical results. 
Our future investigations will concentrate on the resolution of models with a challenging size, the comparison with the product-form approximation, and also on ways to compute efficiently transient measures such as the mean time to depletion. With that objective, we will also investigate whether an approximate analysis through time decomposition is accurate. In a future work, we intent to specify the component interactions of our model by means of high level formalism such as a Markovian process algebra.

\section{References}

1. Akar, N., Oguz, N.C., Sohraby, K.: A novel computational method for solving finite QBD processes. Stoch. Models 16(2), 273-311 (2000)

2. Chao, X., Miyazawa, M., Pinedo, M.: Queueing Networks: Customers, Signals and Product Form Solutions. Wiley (1999)

3. De Cuypere, E., De Turck, K., Fiems, D.: Stochastic modelling of energy harvesting for low power sensor nodes. In: Proc. of QTNA '12 (2012)

4. De Cuypere, E., De Turck, K., Fiems, D.: Performance analysis of a kitting process as a paired queue. Mathematical Problems in Engineering p. 10 (2013)

5. Elhafsi, E.H., Molle, M.: On the solution to QBD processes with finite state space. Stoch. Anal. and Appl. 25(4), 763-779 (2007)

6. Gaver, D.P., Jacobs, P.A., Latouche, G.: Finite birth-and-death models in randomly changing environments. Adv. Appl. Prob. 16(4), 715-731 (1984)

7. Gelenbe, E.: G-networks with triggered customer movement. J. Appl. Prob. 30(3), 742-748 (1993)

8. Gelenbe, E.: A sensor node with energy harvesting. ACM Sigmetrics Perform. Evaluation Review 42(2), 37-39 (2014)

9. Gelenbe, E., Marin, A.: Interconnected wireless sensors with energy harvesting. In: Proc. ASMTA 2015. vol. LNCS 9081, pp. 97-99. Springer Switzerland (2015)

10. Harrison, P., Marin, A.: Product-forms in multi-way synchronizations. Comp. Journal 57(11), 1693-1710 (2014)

11. Jones, G.L., Harrison, P.G., Harder, U., Field, A.J.: Fluid queue models of battery life. In: Proc. of MASCOTS '11. pp. 278-285 (2011)

12. Jones, G.L., Harrison, P.G., Harder, U., Field, A.J.: Fluid queue models of renewable energy storage. In: Proc. of VALUETOOLS '12. pp. 224-225 (2012)

13. Kim, C., Dudin, A., Dudin, S., Dudina, O.: Analysis of an $M M A P / P H_{1}$, $\mathrm{PH}_{2} / \mathrm{N} / \infty$ queueing system operating in a random environment. Int. J. Appl. Math. Comput. Sci. 24(3), 485-501 (2014)

14. Latouche, G., Ramaswami, V.: Introduction to Matrix Analytic Methods in Stochastic Modeling. ASA-SIAM (1999)

15. Latouche, G.: Queues with paired customers. J. Appl. Prob. 18(3), 684-696 (1981)

16. Neuts, M.F.: Matrix-geometric solutions in stochastic models: An Algorithmic Approach. The John Hopkins University Press (1981)

17. Takahashi, M., Osawa, H., Fujisawa, T.: On a synchronization queue with two finite buffers. Queueing Systems 36(1-3), 107-123 (2000)

18. Wimmer, R., Derisavi, S., Hermanns, H.: Symbolic partition refinement with automatic balancing of time and space. Perform. Evaluation 67(9), 816-836 (2010)

19. Ye, J., Li, S.Q.: Folding algorithm: A computational method for finite QBD processes with level-dependent transitions. IEEE Trans. Comm. 42(2/3/4), 625-639 (1994) 